\title{
Survey and Study of various Bug Tracking and Logging Toolkits
}

\author{
Kanaklata \\ M.Tech (CS\&E) \\ World College of Technology and Management \\ Farukhnagar, Gurgaon
}

\author{
Shweta Sharma \\ Assistant Professor \\ World College of Technology and Management \\ Farukhnagar, Gurgaon
}

\begin{abstract}
These days, when project are so wide, bugs or defects do exist as a glitch in the system and they are usually inevitable in software development. A bug might be in either its design or a program's basis code. Massive amount of bugs might be originate in system development. It is comparatively not easy to manage bugs in simple word documents or keep in mind the whole thing in one's brain. Because of this, it becomes very significant to have suitable bug tracking tool. A bug tracking tool handles communication among teams more efficient and every bug and changes are systematically recorded in web based system and hence the approach is scalable. The idea behind this paper is to analyze different bug tracking tools, and the idea is to provide innovative set of selection criteria that provides more gratifying solution, as the industry wants to choose the finest tool among the offered set of tools that will be assisted in fixing and tracking the overall progress of bug fixes.
\end{abstract}

\section{Keywords}

Software Testing, Bug Logging and Tracking Tool, Data storage, Notification Method, web-based application.

\section{INTRODUCTION}

A software bug is a flaw,error, mistake, fault or failurein anapproach or computer program that produces an inappropriate or unpredicted result, or causes it to act in unintended ways. Maximum bugs evolve from mistakes and blunders made by people in moreover a program's design or its source code [1].

By using bug tracking tools, we can keep track of testified software bugs with the assistance of bug tracking tools whereas developer can further enhance software design by making modifications or advancing the software. A bug tracking tool is a software application which is designed to comfort quality assurance and computer programmer keeps track of all the reported software bugs in their task.

Once the bug is superimposed, it is half-tracked through to completion. Users of bug following system is commonly customers, code testers, project team members, management members and code developers. It provides a facility to all or any the users to trace and manage multiple comes at the same time. The testing team will use the bug following system to record problems progress, till such time that a developer might consider the opposite problems.

we all recognize, no software package is ideal, it means most of the software package might need extra module or sweetening of the present module or a number of the module might contain some unnoticed or uncurbed bug that square measure left within the software package from time to time. The bug might get introduced in any part of the SDLC Life cycle, i.e. requirement analysis (RA), design (SD), coding (SC), testing (ST), implementation (SI) and maintenance of the system (SM) [2].

Mostly all the most bug chase systems permits users to insert attachments like MS word and pictures within the comment so developers will reproduce the bug simply and so take applicable action thereon like a shot.

\section{PROBLEM STATMENT}

\subsection{Manually Testing}

Bug coverage consists of tasks like investigation, military operation, testing and debugging throughout the complete method. It's terribly tough to manage problems with a project manually, as a result of many bugs may be found. Developers produce the bugs, however QA team is that the one United Nations agency examine the code and application to get the precise sequence or combination of steps that turns up a mistake.

\subsection{Un-Productive Messaging}

All the system development groups would like effective communication. it's unattainable for anyone to stay all the bugs in one's head or in a very single document like spreadsheets. And, they'll not be able to communicate effectively with each other or with the event team and therefore won't be able to contribute to extend product quality. As a project raises, the primary obstruction that's at risk of be encountered is that solitary one person will amend the computer programme at a amount.

Communication may be an important and deciding issue for the success of a computer code project.

\subsection{Compound System}

Nowadays, sizable amount of comparable bug chase systems obtainable for this purpose; and most of them square measure created with unessential advanced and confusing functions. The flow isn't simple may be a main concern. Most bug chase systems square measure in-built web- based mostly application. Web-based applications will usually be tough to navigate, particularly if they behave additional sort of a sequence of hypertext markup language pages than associate application. Some net applications square measure perpetually reloading pages as a result of responding to user input needs a trip between the net server and also the user's browser. It incurs slowness as involving several reloading of pages and makes the applying tough to figure with.

\section{LITERATURE REVIEW}

SSoftware development project faces multitude of challenges. one in every of those challenges is that the defects found within the project. Defects or bugs area unit issues exist 
within the system and that they area unit usually inevitable in software package development. A software package bug is that the common term accustomed describe a mistake, flaw, mistake, failure, or fault associate degree exceedingly bug or system that produces an incorrect or surprising result, or causes it to behave in unmotivated ways in which. Most bugs arise from mistakes and errors created by individuals in either a program's ASCII text file or its technical style [3].

The presence of bugs has caused the system fail to satisfy customer's cheap expectation of quality. Quality is outlined as meeting the customer's needs and appropriate for the utilization of users. Defect or bug are a few things that doesn't enable the merchandise to satisfy client needs. Defect happens as a result of one thing has not worked as planned. Since that, individuals complete that implementing bug pursuit method is very important so as to seek out, establish and take away bugs or problems inside application and eventually keep the system running properly. Besides, the aim of pursuit bug is to boost the standard of software package products; it's a nonstop truth that fresh written software package packages can contain bugs and it's inevitable. once a system gets a bug, it will cause all kinds of issues. Bugs will cause the system to perform task incorrectly or cause things to occur that ought to not be. Once a product is free to customers, all organizations ought to adhere to some method of managing clients issues for the final word purpose of customer satisfaction.

A useful tool is required to ease the case explicit on top of. Development team desires a scientific approach which may facilitate in pursuit bugs with all data regarding them; such system is thought as bug pursuit system. Development team needs to solve the bugs found so as to form the system works in correct approach. but the quantity of bugs may be found in a very system development life cycle and bug pursuit become difficult [5].

It is more difficult to begin pursuit bugs throughout the software package development phases as a result of software package developers don't seem to be terribly cooperative in pursuit down their faults. They feel that it threatens each their creativeness and skilled ego.

Previously, individuals caterpillar-tracked bug in a very straightforward approach. Some users reported bugs by causation email to connected technical department. this is often rather troublesome to stay track the bug reported because the emails area unit scattered around email application. whereas developers of programs with modest quality will accomplish manual bug pursuit via email, it'd be a supply nightmare for developers of a lot of well-liked software package to try this approach. during this case, the best structured thanks to keep track of alittle variety of bugs is to use a computer programme program, like stand out [4]. the most advantage of this approach is its simplicity and appropriate for small-scale project. Spreadsheets area unit ordinarily offered tools and area unit moderately well documented and users found it easier to use attributable to its options. Besides, basic reports are straightforward enough to provide in computer programme. However, the disadvantages of this approach area unit vital. Most of those area unit constant reasons why individuals use databases instead of spreadsheets. As project grows, the primary drawback that's possible to be encountered is that just one person will edit the computer programme at a time, users cannot modify the computer programme at the same time and it's not economical. One needs to wait till alternative user has finished viewing or piece of writing the computer programme and so solely able to modify it. Another, drawback is that, in contrast to with an honest info, if the pc or server running the computer programme crashes throughout modification of bug, then the info may be inconsistent.

Besides, once the overall variety of bugs within the computer programme grows into thousands, the time taken for the various reports generating will grow considerably. Therefore, mistreatment computer programme for keeping track of bugs ought to be viewed as a short lived live as a result of it'll not scale as a project grows. the reality is that it's comparatively troublesome to manage bugs in straightforward word documents, computer programme or keep in mind everything in one's head once it involves huge scale of project. the knowledge caterpillar-tracked during this approach is troublesome to take care of. Eventually the history of the debugging method or valuable data could be loss. Still, a computer programme is best than associate email folder of reports of potential bugs or a wall jam-packed with sticky notes. however it's not scalable and not counseled for giant scale project. because of this reason, bugs pursuit system is made to assist QA team and development team to stay track of the reported bugs. There area unit several straightforward, affordable bug pursuit systems offered that may scale higher than a computer programme.

\section{TRENDS AND PATTERNS}

Bug tracking database system has been introduced to substitute spreadsheet. In current years, bug tracking systems are extensively deployed in the information technology industry, especially in software development. Hardware and software companies use bug tracking systems throughout the development cycle to track bugs and design issues. Several use it during afterward phases of the product lifecycle to keep track of defect reports and to achieve quality indicators. The biggest advantage is that it contains database that stores every single issues reported by users. Earlier, older bug or defect tracking systems are based on client server and it caused troubles to users at the same time. Users require to install the server, and each user need to install the client software. If external users were involved, it could be problematic because of issues like firewall blocking. It is also not feasible to set up client software in every user's computer.

Nowadays newer systems area unit a lot of doubtless developed in application program based mostly and therefore no shopper code installation is required. A web-based bug trailing system is particularly enticing if users concerned area unit set in several locations and area unit connected through the web. With the help of net access, user will access period data from anyplace within the world, twenty four hours daily, one year a year. A bug trailing system permits users to report a detected bug on to the involved those who will fix it. The system maintains a info that records all facts concerning all defected and reported bugs. the main points a couple of bug could embody its severity, the sudden program behavior, the identity of the user who reported it and identity of skilled who is fixing it. Having a bug trailing system is extraordinarily valuable in code development, and that they area unit used extensively by firms developing code merchandise.

All in all, to trace bugs, several organizations still believe Word documents and surpass spreadsheets, however these ways area unit inefficient and fallible to mention the smallest amount. a decent machine-driven issue-tracking answer ought to contour the method of raising, managing and fixing problems as a result of bug trailing system ought to be helpful code which may be designed to assist code developers keep 
track of reported bug in their alternative code merchandise. Besides, this could be seen joined of the shape of issue trailing system.

Actually bug trailing may be a deceptive term as a result of it's looking on what forms of problems have to be compelled to be half-track. The system will be referred to as as issue trailing as issue may be a broad term to explain most of the forms of tasks would possibly have to be compelled to track once developing associate degree application. the difficulty half-track would possibly embody bugs and feature-changes rather than trailing bugs or defects solely. because it evolved on the time, this method isn't simply designed for internal groups like developers and testers, however additionally for shoppers or alternative finish users to report problems associated with the system. this method enabled clear visibility of the code quality, and improved the design and estimating the analysis and development maintenance effort internally.

\section{SIMILAR AVAILABLE SYSTEMS}

Many firms use bug pursuit system in developing software system product as a result of it's very helpful and valuable. Bug pursuit system is required to store all connected knowledge along as a result of most groups don't adequately maintain their needs and style documents throughout the lifetime of project. Bug pursuit system provides necessary advantages to software system development. Bug pursuit info facilitates clear communication regarding bugs. Well- written, standardized reports tell the story far better than free from emails.

There are various bug pursuit tools obtainable each commercially and as open supply. choose the best bug tracking tool that fulfill the users' demand isn't simple. There are several factors that require to be thought of as follow:

\subsection{Setup of the Application}

Application setup is that the beginning to be done before the system may be utilised. Bugzilla is perhaps the foremost wellknown of the open supply issue-management tools. it's used on several open supply comes as Mozilla, Eclipse, and plenty of UNIX system distributions, and is well-adapted to giant, open comes. however typically software bug pursuit tools may be tough for user to put together. The software bug pursuit tool like Bugzilla sometimes takes an extended time to setup or even not user friendly and supported. The setup method isn't simple and sophisticated. thus users have issue to put in the appliance in server.

Bugzilla may be a tool with AN uninspiring programme and is wealthy in options, however undeniably cumbersome to put in and to take care of. Besides, some supported that Bugzilla is simple to use and quite versatile, however it's terribly tough for a novice to put in and maintain. though in small stages directions are provided, Bugzilla isn't perpetually simple to induce operating. it's annoyed if users take longer time and plenty of labor to setup the appliance so as to form it operating. The setup method ought to be easy and simple.

\subsection{Procedure of Reporting}

The bug reportage method ought to be straightforward and however helpful in trailing bugs. so the steps ought to be simplified and maximising communication effectiveness between users. If the reportage method is sophisticated and not user friendly, users might problem concerning using it and it'll be time overwhelming. Since web-based bug trailing system is wide used today, it incurs some drawback at a similar time. Web-based applications will usually be troublesome to navigate, particularly if they behave a lot of sort of a sequence of markup language pages than Associate in Nursing application. for instance, some net applications square measure perpetually reloading pages once responding to user input needs a trip between the net server and also the user's browser. Eventually the method can be cumbersome and users may later refuse to utilize it to trace the problems associated with system. The quality of the system may cut back the potency of labor and users may feel hard once use the system[6].

Some bug trailing systems square measure on the market with some seldom used options that increase the quality of the reportage method. As a result, the method of reportage bugs or problems into a system should be simple and simple. Bug trailing system is sophisticated or straightforward. Ideally it ought to be straightforward and quick thus on avoid individuals resorting to email or alternative text document. for instance, a bug trailing system known as, "Bug Genie", it's a strong bug trailing system with several options. however the method of reportage bug involves several steps and not uncomplicated. Feedback from users WHO used this technique explicit that configuring comes or adding new problems to the hunter has been created excessively|anexcessively|a very\} troublesome task and it's overly sophisticated bug reportage.

If the bug trailing system is troublesome to use, is timeoverwhelming, or asks plenty of knowledge that the author of an event report sees no want for, use of the system are restricted or the info is also not correct. so an efficient bug trailing systems will improve client satisfaction, raise productivity and cut back downtime[7].

\subsection{Methodology of Messaging}

As we tend to all grasp users might not logged into system all the time, notification may be a vital feature in bug chase system. Notifications area unit very a progress feature as a result of they assist you track and manage bugs. for instance, notifications will keep team members educated concerning vital changes to bugs, like changes in priority or addition of recently additional info. Notifications can even facilitate change the defect management method. for instance, system will apprize development managers once new bugs area unit submitted and developers once bugs area unit assigned to them. Most systems like "Flyspray" and "BugTracker.NET" use email solely to apprize users once there area unit updates within the system however there's an opening that emails may be blocked by spam filter and not delivered to the selected recipients. during this case, email notification may not be a reliable channel to inform users [8].

\section{EXISTING BUG TRACKING TOOLS AND THEIR DRAWBACKS}

In this section, we tend to offer a summary of existing bug following tools and that we criticize the present solutions from the purpose of their usability

\subsection{BugZilla}

Bugzilla is incredibly widespread, actively maintained and free bug following system, used and developed beside Mozilla, giving it respectable credibleness. Bugzilla is predicated on Perl and once it's established, it looks to form its users pretty happy. It's not extremely customizable, however during a odd manner, that will be one amongst its features: Bugzilla installations tend to appear just about a similar where they're found, which suggests several developers area unit 
already acquainted with its interface and can feel they're in acquainted territory. Bugzilla encompasses a system which will send you, another user, or a gaggle that you just specify the results of a specific search on a schedule that you just specify. Bugzilla encompasses a terribly advanced reportage systems and you'll produce differing types of charts together with line graph, bar chart or chart.

\subsection{Mantis BT}

Mantis could be a free web-based bug following system. it's written within the PHP scripting language and works with MySQL, MS SQL, and PostgreSQL databases and a webserver. dictyopterous insect is put in on Windows, Linux, mackintosh OS and OS/2. virtually any applications programme ought to be ready to perform as a shopper. it's discharged underneath the terms of the antelope General Public License (GPL). the most grievance is its interface that doesn't meet electronic equipment standards. On the opposite hand, is straightforward to navigate, even for inexperienced users. There not exists some advanced options like charts and reports. In short, the complete system is sloppily done, there area unit lots of bugs and really very little practicality.

\subsection{BugTracker.NET}

BugTracker.NET could be a free, ASCII text file, web-based bug huntsman or client support issue huntsman written victimization ASP.NET, C\#, and Microsoft SQL Server specific. BugTracker.NET is straightforward to put in and learn the way to use. once you first install it, it's terribly straightforward to setup and you'll begin victimization it at once. Later, you'll amendment its configuration to handle your desires. it's a really intuitive interface for generating lists of bugs. it's two very helpful options. 1st of them could be a screen capture utility that allows you to capture the screen, add annotations and post it as bug in barely a number of clicks. The second feature is that the incontrovertible fact that it will integrate along with your Subversion repository so you'll associate file revision checkins with bugs.

\subsection{FlySpray}

Flyspray could be a web-based bug following system written in PHP. Flyspray is free software package, discharged underneath the final Public License. This primarily implies that you'll get Flyspray and use it freed from charge. The ASCII text file is out there, and everybody area unit welcome to change it to suit their desires. Its websites describe it as "uncomplicated", and therefore the list of options includes: multiple information support (currently MySQL and PGSQL), multiple comes, 'watching' tasks, with notification of changes (via email or Jabber), comprehensive task history, CSS teaming, file attachments, advanced search options, RSS/Atom feeds, wiki and plaintext input, voting, dependency graphs.

\subsection{RedMine}

Redmine could be a versatile internet-based project management web application. Written victimization Ruby on Rails framework, it's cross-platform and cross-database. Redmine is open supply and discharged underneath the terms of the antelope General Public License. Redmine is versatile issue following system. you'll outline your own statuses and issue sorts. It supports multiple comes and subprojects. every user will have a unique role on every project. Interface is incredibly straightforward, intuitive and simple to navigate. Shortly, this is often excellent product and our recommendation.

\subsection{RedMine}

Bug-Track is web-based defect and bug following software package permits you to document, manage and assign all of your bugs and tasks and empowers you to prepare your bugs, defects or problems into distinct comes. It will run on nearly any web-server like Microsoft, Linux, Unix, etc... Since it's Associate in Nursing business application it's expected that it's higher than alternative free merchandise. however it isn't true. He has nothing new and higher than alternative free bug following systems. One higher issue is incontrovertible fact that he have a lot of intuitive interface then others which is his solely profit.

\subsection{BugZero}

Bugzero could be a web-based bug, defect, issue and incident following software package. Its single code base supports each Windows and UNIX operating system (based on JavaTM) and supports information systems together with Access, MySQL, SQL Server, Oracle, and etc. Bugzero is custom for software package bug following, hardware defect following, and facilitate table client support issue and incident following. Bugzero have intuitive interface however he lacks type options. the most downside is that the incontrovertible fact that Bugzero is Associate in Nursing business product and you'll realize far better product at no cost.

\section{FUTURE SCOPE}

When a user submits a bug report, he's asked several questions: what's the name of the product? during which plugin/component? what's the Build ID? what's the bug about? What area unit the steps to reproduce the bug? Any extra information?

However, the initial info provided during a bug report typically|isusually|is commonly\} incomplete and developers often have follow-up questions: does one have flash installed? Is there any screenshot? obtaining replies by users takes time (often weeks) and slows down the progress on open bugs. And this makes the full method sluggish and slows down the method of bug pursuit.

The advantages of the improved set of criteria can offer user higher info concerning current bug. this may result in quicker locating of this defect and to the quicker reply to it.

\section{CONCLUSION}

Existing bug trailing systems don't effectively accumulate all of the knowledge required by developers. while not this info developers cannot resolve bugs in an exceedingly timely fashion and then we have a tendency to believe that enhancements to the method issue trailing systems collect info are required.

We summarized criteria that are employed in electronic equipment bug trailing systems. Such criteria usually doesn't provide acceptable ends up in describing bug. So, we have a tendency to projected an improved set of criteria which will provide far more satisfying resolution for the present system.

This work are often vital to the designers of the longer term bug and defect trailing systems. they ought to grasp importance of choice criteria for describing bug, as a result of a well delineate bug are easier to be trace and solved

\section{REFERENCES}

[1] Prof Dr. Muhammad YounusJaved and HufsaMohsin, 2012 on "An Automated Approach for Software Bug Classification" in Complex, Intelligent and Software Intensive Systems (CISIS) 2012 page 414-419. 
[2] V.B Singh, Krishna Kumar Chaturvedi on "Bug Tracking and Reliability Assessment System" international Journal of Software Engineering and Its Applications Vol. 5 No. 4, October, 2011 page 17-30.

[3] D'Ambros, M. Lugano Univ., Lugano Lanza, M.; Pinzger, M.on "A Bug's Life" Visualizing a Bug in Visualizing Software for Understanding and Analysis,2007Page(s): 113-120

[4] Sascha, Rahul Premraj and Thomas Zimmermann on 'Towards the Next Generation of Bug Tracking Systems' in Visual Languages and Human-Centric Computing, 2008 on Page(s):82-85

[5] Catherine V. Stringfellow, DileepPotnuri on "Analysis of Open Source Defect Tracking Tools for Use in Defect Estimation" Software Engineering Research and Practice 2005: page no.296-301
[6] Davidson, J.L., Mohan, N.; Jensen, C. on "Coping with Duplicate Bug Reports in Free/Open Source Software Projects" in Visual Languages and Human-Centric Computing (VL/HCC), 2011 IEEE Symposium on Page(s): $101-108$

[7] Liguo Yu, Stephen R. Schach, Kai Chen on "Measuring the Maintainability of Open-Source Software" in International Symposium on Empirical Software Engineering, 2005.

[8] Thomas Zimmermann, Rahul Premraj, Nicolas Bettenburg, Sascha, Adrian Schrter, and Cathrin Weiss on What Makes a Good Bug Report? In international symposium on Foundations of software engineering page 308-318 